\title{
Letramento funcional em saúde de idosos hipertensos e diabéticos atendidos na Estratégia Saúde da Família
}

Functional health literacy among hypertensive and diabetic elderly assisted by the Family Health Strategy Alfabetización en la salud de ancianos hipertensos y diabéticos atendidos en la Estrategia Salud de la
Familia

Helenice de Moura Scortegagna ${ }^{1}$ (1)

Paulo Cassiano Simor dos Santos ${ }^{1}$ (1) Maria Izabel Penha de Oliveira Santos ${ }^{1,2}$ (1)

Marilene Rodrigues Portella ${ }^{1}$ (B)

1. Universidade de Passo Fundo, Instituto de Ciências Biológicas, Programa de PósGraduação em Envelhecimento Humano. Passo Fundo, RS, Brasil.

2. Universidade do Estado do Pará, Departamento de Enfermagem. Belém, PA, Brasil.

Autor correspondente:

Helenice de Moura Scortegagna.

E-mail: helenice@upf.br.

Recebido em 12/06/2020.

Aprovado em 31/03/2021.

\section{Resumo}

Objetivo: avaliar o letramento funcional em saúde de idosos hipertensos e diabéticos adscritos a Estratégia Saúde da Família. Método: estudo transversal, desenvolvido com 78 idosos acometidos por Diabetes Mellitus tipo Il e Hipertensão Arterial Sistêmica, residentes em município do interior do estado do Rio Grande do Sul. Para a coleta dos dados foi aplicado questionário com informações sociodemográficas e de saúde e o instrumento S-TOFHLA. Para análise dos dados foram utilizados os testes qui quadrado de Pearson; Medidas de Tendência Central (média e mediana) e dispersão, $a=5 \%$ e $p \leq 0,05$. Resultados: o nível de letramento funcional em saúde demonstrou-se inadequado em $55,1 \%(\mathrm{~N}=43)$ dos participantes, e limítrofe em $30,8 \%(\mathrm{~N}=24)$ Identificou-se o predomínio de hipertensão arterial e tempo de tratamento superior a 10 anos. Os resultados mostraram associação entre baixa escolaridade e inadequado letramento funcional em saúde $(p<0,002)$. Conclusão e implicações para a prática: o letramento funcional em saúde inadequado em idosos portadores de doenças crônicas, muitas vezes responsáveis pelo seu autocuidado, pode contribuir para agravos na condição de saúde e doença dessa população, sendo relevante seu reconhecimento para o estabelecimento de estratégias e ações que visem melhores resultados na produção do cuidado.

Palavras-chave: Envelhecimento; Doença Crônica; Saúde do Idoso; Competência em Informação; Estratégia Saúde da Família.

\section{Abstract}

Objective: to assess functional health literacy among hypertensive and diabetic elderly individuals enrolled in the Family Health Strategy. Method: cross-sectional study conducted among 78 elderly individuals with type 2 Diabetes Mellitus and Systemic Arterial Hypertension, residing in a city in the interior of Rio Grande do Sul, Brazil. Data were collected using a questionnaire addressing sociodemographic and health information and the S-TOFHLA. Data analysis included Pearson's Chi-square, Central Tendency Measures (mean and median), and measures of dispersion, $a=5 \%$ and $p \leq 0.05$. Results: inadequate functional health literacy was found among $55.1 \%(\mathrm{~N}=43)$ of the participants, while $30.8 \%(\mathrm{~N}=24)$ presented marginal literacy skills. Hypertension was the most frequent disease with duration of more than 10 years. The results show an association between schooling and inadequate functional health literacy $(p<0.002)$. Conclusion and implications for practice: inadequate health literacy among elderly individuals with chronic diseases who are often responsible for self-care may worsen this population's health conditions and diseases. Thus, identifying literacy levels among these individuals is vital to devise strategies and implement actions intended to promote care.

Keywords: Aging; Chronic Disease; Health of the Elderly; Information Literacy; Family Health Strategy.

\section{Resumen}

Objetivo: evaluar la alfabetización funcional en la salud de ancianos hipertensos y diabéticos inscritos en la Estrategia Salud de la Familia. Método: estudio transversal, desarrollado en 78 ancianos afectados por Diabetes Mellitus tipo II e Hipertensión Arterial Sistémica, residentes en un municipio del interior del estado de Rio Grande del Sur. Para la recolección de datos fue aplicado un cuestionario con informaciones sociodemográficas y de salud y el instrumento S-TOFHLA. Para el análisis de los datos fueron utilizadas, las pruebas Chi-cuadrado de Pearson, Medidas de Tendencia Central (media y mediana) y de dispersión; con $\mathrm{a}=5 \%$ y $\mathrm{p} \leq 0,05$. Resultados: el nivel de alfabetización funcional en salud se demostró inadecuado en $55,1 \%$ ( $\mathrm{N}=43$ ) de los participantes, y limítrofe en $30,8 \%(\mathrm{~N}=24)$. Se identificó el predominio de hipertensión arterial y del tiempo de tratamiento superior a 10 años. Los resultados mostraron asociación entre baja escolaridad e inadecuada alfabetización funcional en salud ( $p<0,002)$ Conclusión e implicaciones para la práctica: la alfabetización funcional en salud inadecuada en ancianos afectados por enfermedades crónicas, muchas veces responsables, por su propio autocuidado, puede contribuir para agravar la condición de la salud y de las enfermedades de esa población, siendo relevante su reconocimiento para el establecimiento de estrategias y acciones que tengan por objetivo obtener mejores resultados en la producción del cuidado.

Palabras clave: Envejecimiento; Enfermedad Crónica; Salud del Anciano; Alfabetización Informacional; Estrategia de Salud Familiar. 


\section{INTRODUÇÃO}

A longevidade humana tem alterado, significativamente, o perfil demográfico nas populações em todo o mundo, assim como tem refletido no comportamento epidemiológico das doenças, com significativo aumento das situações patológicas crônicas. Embora considere-se a longevidade como uma importante conquista da humanidade, tal realidade somente pode ser exaltada quando os anos vividos a mais são gozados com qualidade, em um cenário no qual o idoso possa gerir com autonomia a própria vida e saúde. ${ }^{1}$ Caso contrário, o processo de envelhecer poderá repercutir negativamente, com diversas implicações, tanto para o ser "envelhecente", quanto para a família e a sociedade.

O Brasil tem vivenciado a transição demográfica de forma acelerada, pois se observa na faixa etária acima dos 60 anos um movimento inverso a faixa etária dos zero aos nove anos, ao que projeções para 2040 estimam que, vão representar $23,4 \%$ e $10,1 \%$, respectivamente, da população total. ${ }^{2}$ Com relação à transição epidemiológica, a heterogeneidade do segmento idoso configura um desafio aos serviços de saúde na medida em que pode representar significativa probabilidade da ocorrência de eventos patológicos, vulnerabilidades e fragilidades. $\mathrm{O}$ enfrentamento das doenças crônicas não transmissíveis, que acometem especialmente o segmento de maior idade da população, se configura como uma das prioridades públicas, segundo o Plano de Ações Estratégicas para 2011-2022, no Brasil, considerando que as doenças crônicas não transmissíveis como hipertensão arterial, diabetes, infarto, acidente vascular cerebral, câncer e doenças respiratórias crônicas têm correspondido a aproximadamente $70 \%$ das causas de mortes. ${ }^{3}$ De acordo com essa publicação, o estrato mais atingido seria o de baixa renda e escolaridade. Ressalva-se que, um fator de destaque e que pode refletir na qualidade de vida e saúde dos longevos diz respeito ao baixo nível educacional. Dados oriundos do Instituto Brasileiro de Geografia e Estatística refletem uma realidade preocupante ao destacar que a taxa de analfabetismo é maior nos grupos de idade mais elevada, alcançando, em 2015, 22,3\% entre as pessoas de 60 anos ou mais. ${ }^{4}$

Outro importante indicador educacional, avaliado pelo Instituto Paulo Montenegro e a Associação Civil Ação Educativa, é o Alfabetismo Funcional, também denominado letramento funcional, que corresponde a condição daqueles indivíduos que não têm as competências necessárias para atender as demandas e tarefas do cotidiano, pessoal e profissional, mesmo sabendo ler e escrever algo simples. A partir da mensuração desse indicador na população brasileira, o estudo realizado por essas organizações explicita que, o percentual classificado como analfabeto funcional, apresentou um novo aumento no seu patamar, em 2018 (29\%), comparado aos resultados obtidos de 2009-2015 (27\%); os indivíduos na faixa etária entre 50 e 64 anos da população são os que apresentam os maiores números nessa classificação (53\%). Chama atenção que o perfil de escolaridade, entendido como o tempo de permanência do indivíduo na escola e sua progressão no ensino formal, se apresenta significativamente relacionado ao alfabetismo funcional, mas não de forma uniforme e linear, pois uma proporção expressiva de pessoas com ensino médio e superior não atingiram o grupo mais elevado da escala de alfabetismo funcional. ${ }^{5}$

Ampliando o conceito de letramento funcional, as competências básicas de leitura, escrita e habilidades matemáticas, aplicadas no contexto de saúde, são reconhecidas como letramento funcional em saúde, e envolvem uma série de fatores sociais e individuais, incluindo conhecimento cultural e conceitual, habilidade de ouvir, falar, aritmética, escrita e leitura. ${ }^{5}$ Essas habilidades cognitivas e sociais, descritas por esse constructo, tornam-se ferramentas importantes para que o indivíduo acometido por uma doença possa participar do planejamento e implementação da terapêutica, sendo de vital importância para o desfecho de sucesso do tratamento. ${ }^{6}$ Nessa direção, as competências necessárias para obter, processar e agir de acordo com as informações e conhecimentos sobre saúde podem estar comprometidas frente à dificuldade em ler e escrever. ${ }^{7}$

As competências básicas de letramento funcional em saúde, quando inadequadas, poderão ocasionar dificuldades para as pessoas quanto à compreensão das instruções, da leitura de rótulos; de receitas e bulas de medicamentos, necessários para adesão ao esquema terapêutico, bem como para contemplar ações de prevenção de doenças e promoção da saúde. .10 $^{8}$ Contudo, quando um idoso vivencia uma situação que requer cuidados de saúde prolongados e apresenta baixa escolaridade e/ou o analfabetismo, tais condições podem afetar e repercutir de forma negativa no manejo da doença, pois a escolaridade tem se revelado como um dos principais fatores explicativos da condição de alfabetismo. ${ }^{7}$ Essa constatação já representava, há dez anos, uma preocupação da Organização Mundial da Saúde que enfatizava que menor instrução e analfabetismo estão associados a maiores riscos de deficiência e morte no processo de envelhecimento. ${ }^{11}$ Portanto, a compreensão de que a alfabetização deficiente pode afetar à saúde das pessoas, limitando o seu desenvolvimento pessoal, social e cultural, resultou na identificação do Letramento Funcional em Saúde como um determinante social da saúde das pessoas. ${ }^{12}$

Os indivíduos com baixo letramento funcional em saúde terão habilidades menos desenvolvidas para atuar sobre a informação recebida e menor sensibilidade à educação em saúde, podendo assumir hábitos de risco para a sua saúde ou de sua família. ${ }^{13}$ No que se refere a gestão das doenças crônicas, muitos são os desafios, considerando o alto nível de complexidade da doença e de autocuidado necessários, tal qual a necessidade da compreensão da doença e tratamento. Diante desse cenário, o déficit na capacidade para interpretar e utilizar informações em saúde pode repercutir na manutenção da doença crônica. ${ }^{14}$ Importante destacar que o letramento em saúde molda o comportamento e as escolhas das pessoas para saúde e bem-estar, ${ }^{7,13}$ contudo configura uma construção complexa que depende tanto da capacidade individual de se comunicar, como das exigências impostas pela sociedade e sistema de saúde. Representa uma estratégia de empoderamento fundamental para ampliar o controle da população sobre a sua saúde, sua capacidade de buscar informações e assumir a 
responsabilidade sobre as questões de saúde. Corroborando com esta compreensão, o Institute of Medicine ${ }^{15}$ e o National Academy of Medicine ${ }^{16}$ destacam que o letramento em saúde é multidimensional, resultante de diferentes demandas e de uma complexa convergência entre educação, fatores sociais e serviços de saúde.

No que se refere ao desenvolvimento do letramento funcional em saúde, as ações de educação em saúde devem apresentar objetivos focados na melhoria dos conhecimentos sobre os riscos à saúde, bem como aos serviços de saúde e à adesão às prescrições dos profissionais, pelos indivíduos, representando a ligação entre as habilidades de letramento, o contexto da saúde e a capacidade individual. ${ }^{17} \mathrm{~A}$ partir da compreensão de que o letramento funcional em saúde não se restringe a uma habilidade única de letramento em ambiente clínico, mas que se refere à aplicação prática de diversas competências cognitivas e não cognitivas no cotidiano da vida, pode-se afirmar que a avaliação desse indicador na população se configura como um caminho que permite o aprimoramento de ações em prol da promoção de saúde e da prevenção de doenças crônicas não transmissíveis, no que diz respeito ao planejamento, execução e avaliação. ${ }^{15,18}$ Dessa forma, para que um indivíduo possa exercer as ações pertinentes ao campo da saúde, a compreensão e a aplicação das informações em saúde, figuram elementos essenciais para o estabelecimento das condições necessárias para a manutenção de uma boa saúde. Ao tratar-se de um indivíduo idoso, tais obstáculos podem apresentar impactos mais significativos, uma vez que além dos deficits cognitivos e sensoriais que podem estar presentes, e dos baixos níveis de alfabetização da população idosa brasileira, um inadequado letramento funcional em saúde pode repercutir diretamente na possibilidade em manter a promoção das condições de vida e de saúde. Nesse sentido, considerando que, em idosos, pode ser um preditor de risco para a saúde, torna-se relevante sua avaliação. Portanto, teve-se como questão norteadora, qual o nível de letramento funcional em saúde de idosos hipertensos e diabéticos adscritos a Estratégia Saúde da Família? O presente estudo teve por objetivo avaliar o letramento funcional em saúde de idosos hipertensos e diabéticos adscritos a Estratégia Saúde da Família.

\section{MÉTODO}

Trata-se de uma pesquisa transversal, exploratória, de abordagem quantitativa, desenvolvida com idosos acometidos por Diabetes Mellitus tipo II e Hipertensão Arterial Sistêmica, atendidos pela Estratégia Saúde da Família em duas unidades básicas de saúde, vinculadas à Secretaria Municipal de Saúde, em município do interior do estado do Rio Grande do Sul.

A população do estudo foi composta por 78 indivíduos idosos, de ambos os sexos, cadastrados no extinto Sistema de Cadastramento e Acompanhamento de Hipertensos e/ou Diabéticos - HIPERDIA do Sistema Único de Saúde, no ano de 2015. Para a composição da amostra foram identificados os idosos utilizando-se para o cálculo amostral a fórmula para populações finitas. ${ }^{19}$ Assim, a partir da população de referência
(113 idosos), se estabeleceu uma amostra de 88 idosos. Foram, posteriormente, selecionados conforme critérios de inclusão previstos neste estudo: indivíduos com 60 anos ou mais, em tratamento/acompanhamento para Diabetes Mellitus tipo II e Hipertensão Arterial Sistêmica, cadastrados no sistema HIPERDIA/SUS, a partir de bom resultado obtido na aplicação da Mini Avaliação Cognitiva, que incluiu o teste do desenho do relógio, e do resultado no teste do sussurro (Teste de Whisper), que avaliou a acuidade auditiva, e do teste de acuidade visual, pelo Cartão de Jaeger. ${ }^{20}$ Excluíram-se da amostra os idosos não alfabetizados, portadores de deficit visual e/ou auditivo e os que apresentaram dificuldade motora fina das mãos que impossibilitariam a realização dos testes (10 idosos). A coleta de dados foi realizada no período de maio a julho de 2015, no domicílio dos idosos, em data e horário agendados antecipadamente, após contato prévio com os enfermeiros e agentes comunitários de saúde das Estratégias Saúde da Família, com a colaboração de graduandos de enfermagem treinados.

Os instrumentos de coleta de dados utilizados foram um questionário estruturado que continha informações referentes às características sociodemográficas e de saúde e o teste de alfabetização funcional para adultos na área da saúde - Test of Functional Health Literacy in Adults - TOFHLA (versão breve), ${ }^{21}$ traduzido e aplicado no Brasil. ${ }^{22}$ A versão reduzida do teste, denominada de Short TOFHLA (S-TOFHLA), foi criada objetivandose a redução do tempo de aplicação do mesmo. O S-TOFHLA é composto por 13 frases, com 36 lacunas em branco, das quais o indivíduo deverá escolher entre quatro palavras a que irá completar a frase. Composto também por quatro questões relacionadas à compreensão numérica. Os escores do teste permitem classificar o letramento funcional em saúde como inadequado, para pontuações entre zero e 53 pontos, limítrofe entre 54 e 66 pontos, e adequado para escores que variam entre 57 e 100 pontos. As respostas fornecidas ao questionário e o resultado do S-TOHFLA foram analisadas por meio do programa estatístico Statistical Package for Social Sciences (versão 18.0) e utilizados os testes qui-quadrado de Pearson, Medidas de Tendência Central (média e mediana) e dispersão, com nível de significância de $5 \%$.

O estudo seguiu as diretrizes da Resolução do Conselho Nacional de Saúde n 466/12 sobre a participação de pessoas em pesquisas, como assinatura do Termo de Consentimento Livre e Esclarecido e a garantia de sigilo, anonimato e respeito aos valores do sujeito. O projeto foi aprovado pelo Comitê de Ética em Pesquisa da Universidade de Passo Fundo (Parecer 631.431; CAAE 30273514.1.0000.5342) e consentido pela Secretaria Municipal de Saúde de Passo Fundo.

\section{RESULTADOS}

Em relação às características sociodemográficas, dos 78 idosos participantes, $30,8 \%(\mathrm{~N}=24)$ eram do sexo masculino e $69,2 \%(N=54)$ do sexo feminino. A idade variou entre 60 e 87 anos, predominando a faixa etária de 60 a 69 anos (60,3\%), seguido dos indivíduos com idade entre 70 e 79 anos (34,6\%) e $5 \%$ entre 80 e 89 anos de idade; $78 \%(N=61)$ da amostra se 
declararam casada, $52 \%(\mathrm{~N}=41)$ procedentes do Município de Passo Fundo, e $82 \%$ ( $\mathrm{N}=64)$ dos indivíduos relataram possuir alguma renda mensal. No que diz respeito à escolaridade, metade dos sujeitos $(\mathrm{N}=39)$ declararam possuir de um a quatro anos de estudo, 39,7\% ( $\mathrm{N}=31)$ declararam ter estudado entre quatro a oito anos e pouco mais de $10 \%(\mathrm{~N}=8)$ da amostra alegou ter mais de oito anos de escolaridade. Quanto ao diagnóstico principal, $61,5 \%(\mathrm{~N}=48)$ dos indivíduos possuíam diagnóstico de Hipertensão Arterial Sistêmica, 5,1\% (N=4) de Diabete Mellitus e 33,3\% ( $\mathrm{N}=26)$ sujeitos eram acometidos por ambas as patologias. $\mathrm{O}$ tempo de tratamento das doenças mais referido foi acima de 10 anos por $37,2 \%(\mathrm{~N}=29)$, e de um a cinco anos por $34,6 \%(\mathrm{~N}=27)$, seguido de $28,2 \%(\mathrm{~N}=22)$ entre cinco e 10 anos. Em relação aos hábitos de vida, a maioria declarou não ser tabagista e não consumir bebida alcoólica.

No que se refere às questões sobre a compreensão das informações repassadas pelos profissionais da equipe de saúde, aproximadamente $71,8 \%(\mathrm{~N}=56)$ dos sujeitos referiram a compreensão e $26,9 \%$ ( $N=21)$ expressaram a não compreensão das mesmas. $O$ hábito de leitura foi mencionado por $47,4 \%$ $(\mathrm{N}=37)$ dos participantes, sendo que $24,4 \%(\mathrm{~N}=19)$ referiram não ter o costume. Mediante a aplicação do teste para avaliação do letramento funcional em saúde, $55,1 \%(\mathrm{~N}=43)$ dos participantes demonstraram nível de letramento funcional em saúde inadequado, e os demais foram classificados com escores de limítrofe $30,8 \%$ $(\mathrm{N}=24)$ e de adequado 14,1\% ( $\mathrm{N}=11)$, conforme Tabela 1.

Com relação à associação entre letramento funcional em saúde e escolaridade, os dados evidenciam significância $(p<0,002)$, destacando que no grupo avaliado quanto menor a escolaridade, menores os escores do letramento funcional em saúde, conforme Tabela 2.

Tabela 1. Comportamento do Letramento Funcional em Saúde dos idosos da amostra. Passo Fundo, Rio Grande do Sul, Brasil, 2015. $(\mathrm{N}=78)$

\begin{tabular}{lcc}
\hline \multicolumn{1}{c}{ Variáveis } & F & $\%$ \\
\hline Letramento Funcional em Saúde & & \\
Inadequado & 43 & 55,1 \\
Limítrofe & 24 & 30,8 \\
Adequado & 11 & 14,1 \\
\hline
\end{tabular}

\section{DISCUSSÃO}

Com relação à caracterização sociodemográfica da amostra, observou-se a predominância do sexo feminino, destacando uma realidade mundial demonstrada em estudos sobre o envelhecimento humano. ${ }^{18,22,23,24}$ Esses resultados estão em consonância com o que o IBGE explicita: a maior prevalência das mulheres na pirâmide demográfica brasileira, especialmente em maior concentração nos grupos de 60 anos ou mais. ${ }^{4}$

Essa investigação centrou em sujeitos acima de 60 anos, havendo predomínio da faixa etária de 60-69 anos, diferenciando este estudo dos realizados em âmbito nacional com o instrumento TOFHLA. ${ }^{18,22}$ Há de se levar em conta, no entanto, estudo que teve por objetivo a adaptação transcultural de instrumento canadense que analisa alfabetização em saúde das pessoas idosas, health literacy, ${ }^{25}$ que mostra compreensão quanto a possibilidade de agravos importantes da saúde, com presença de declínios cognitivos, sensoriais e funcionais, carecer de um olhar mais atento no que se refere às habilidades de letramento voltadas ao contexto da saúde no envelhecimento.

Em relação ao diagnóstico principal, a hipertensão arterial sistêmica apresentou-se como a condição de maior predominância na amostra, com mais da metade dos idosos acometidos por essa patologia. Este resultado se encontra consoante à presença da hipertensão arterial evidenciada na amostra por domicílios, ${ }^{4}$ que teve a prevalência de $24,2 \%$ para as mulheres e $18,3 \%$ para os homens. Apesar de não ser uma consequência natural do processo do envelhecimento, sabe-se que tem correlação direta com a idade. ${ }^{20}$

Em relação ao tempo de tratamento, os idosos pesquisados relataram, em sua maioria, possuir tempo superior a uma década de tratamento. Essa constatação pode sugerir que, quanto maior o tempo de tratamento, maiores seriam os conhecimentos em torno da doença e esquema terapêutico. No entanto, também é possível sugerir indolência frente à necessidade de cuidados complexos e contínuos, próprios das doenças crônicas. Nesse sentido, estudo realizado em uma unidade básica distrital de saúde, do Município de Ribeirão Preto, no qual foram entrevistados 123 usuários com Diabetes Mellitus tipo II, com objetivo de relacionar o conhecimento e a atitude desses usuários com a escolaridade e o tempo da doença, demonstrou que a escolaridade e o tempo da doença se mostraram estatisticamente significantes para a

Tabela 2. Comportamento do Letramento Funcional em Saúde conforme a escolaridade dos idosos que participaram do estudo. Passo Fundo, Rio Grande do Sul, Brasil, 2015. ( $\mathrm{N}=78$ )

\begin{tabular}{|c|c|c|c|c|}
\hline Escolaridade & & Letramer & em Saúde & \\
\hline \multirow{2}{*}{ (em anos) } & Inadequado & Limítrofe & Adequado & \multirow{2}{*}{ Total } \\
\hline & $f(\%)$ & $f(\%)$ & $f(\%)$ & \\
\hline $1-4$ & $26(60,5)$ & $15(34,9)$ & $2(4,7)$ & 43 \\
\hline $4-8$ & $11(45,8)$ & $11(45,8)$ & $2(8,3)$ & 24 \\
\hline $8 a+$ & $2(18,2)$ & $5(45,5)$ & $4(36,4)$ & 11 \\
\hline
\end{tabular}

*p valor $=0,002$. Teste qui-quadrado de Pearson. Tabela elaborada pelos autores 
aquisição do conhecimento e prontidão para ao autocuidado em diabetes. Quanto à atitude, avaliou que a prevalência de adesão ao tratamento apresentou relação inversa ao tempo de diagnóstico, o que remete a possibilidade de maior risco de complicações em virtude de controle metabólico inadequado. ${ }^{26}$

No que se refere à escolaridade dos idosos participantes deste estudo, pode-se afirmar que metade da amostra referiu baixa escolaridade, entre um e quatro anos de estudo. Os demais idosos alegaram escolaridade entre quatro a oito anos e uma pequena parcela confirmou possuir mais de oito anos de estudo. A baixa escolaridade confirma achados em estudos que também evidenciaram parcela significativa de sujeitos com menos de oito anos de estudo. ${ }^{18,27-29}$

Em relação à avaliação do letramento funcional em saúde, destacou-se o elevado contingente de idosos com inadequado letramento funcional em saúde, com mais de $55 \%$ dos sujeitos avaliados, seguido do escore limítrofe, o que se encontra em conformidade com a literatura. ${ }^{22,30,31} \mathrm{Em}$ estudo realizado com 312 participantes saudáveis, na cidade de São Paulo, com idades que variaram entre 18 e mais de 65 anos (média 47,3 3 16,8), pode-se avaliar que, em $32,4 \%$ dos sujeitos, o desempenho no letramento funcional em saúde foi considerado inadequado. Entre os idosos com mais de 65 anos, 38,3\% demonstraram déficits de alfabetização funcional, com desempenho inadequado ou limítrofe no instrumento, e escores marginais em 13,3\%, atestando que metade da amostra, nessa faixa etária, não apresentava escores adequados no teste de alfabetização funcional. ${ }^{22}$ Vale ressaltar, que os idosos classificados com nível de letramento funcional em saúde limítrofe, aproximadamente um terço neste estudo, poderiam apresentar escores próximos do inadequado.

Neste estudo, houve associação entre o letramento funcional em saúde e escolaridade, pelo teste qui-quadrado de Pearson $(p<0,002)$, ou seja, os resultados demonstraram que, quanto menor a escolaridade, menores os escores de letramento funcional em saúde apresentado pela amostra, demonstrando maiores dificuldades de compreensão das informações evidenciadas no contexto de saúde. Tal achado vem ao encontro do apresentado em estudos realizados, nos quais foi possível verificar que, quanto maior a faixa etária dos sujeitos, maior a constatação dos participantes com limitado letramento funcional em saúde. ${ }^{32,33} \mathrm{No}$ entanto, a escolaridade por si só não é um indicador totalmente fidedigno das habilidades, tanto de compreensão quanto de leitura, e não pode ser sempre utilizada como critério para inferir os níveis de letramento em saúde. ${ }^{5,34}$

A discussão em torno dos instrumentos, tal qual a associação direta com as habilidades de leitura e escrita dos usuários dos serviços de saúde, ainda é pauta de discussões por estudiosos do letramento em saúde, não cabendo aqui discutir tais instrumentos e sua aplicabilidade..$^{35}$ Contudo, o S-TOFHLA (versão brasileira), mostrou-se ser uma ferramenta prática para a triagem de indivíduos que apresentam analfabetismo funcional no contexto dos cuidados de saúde, tendo a utilidade do instrumento centrada na identificação das pessoas com maiores necessidades de cuidados especiais. ${ }^{31,34}$
A partir da compreensão acerca da demanda que emerge da gestão do autocuidado, foi realizado estudo com idosos internados no serviço de emergência do Hospital das Clínicas de Porto Alegre, porque, segundo os autores, conhecer o perfil desses idosos subsidiaria intervenções em saúde pelas equipes, tanto da atenção primária como hospitalar, fortalecendo o cuidado integral, por meio de um espaço que permita construir e/ou articular saberes no sentido de atender as especificidades do segmento idoso da população. ${ }^{36}$ Nesse prisma, infere-se que o profissional da saúde representa figura importante para o fortalecimento do letramento funcional em saúde como ferramenta de rastreio dos indivíduos com dificuldades de compreensão e aplicação das recomendações em saúde, com avaliações constantes e capacitação dos envolvidos na atenção ao idoso, principalmente ao acometido por agravos crônicos à saúde. ${ }^{25,37}$

Diante desse contexto, acredita-se que existe a necessidade urgente dos profissionais da saúde estarem atentos para reconhecer e avaliar o idoso, incluindo o letramento funcional em saúde na avaliação multidimensional. Essa proposição relaciona-se ao fato de os profissionais apresentarem compreensão limitada sobre o nível de letramento funcional em saúde para, a partir da sua identificação, poder oferecer recomendações e serviços que atendam às reais necessidades do indivíduo. ${ }^{38} \mathrm{Nessa}$ perspectiva, considera-se importante os profissionais estarem capacitados para levar em consideração que alguns dos usuários não detêm o mesmo conhecimento de outros (que usualmente tem maior aproximação com textos e trabalhos na área da saúde) e assegurar o entendimento acerca das informações e recomendações oportunizadas por ocasião do atendimento. ${ }^{39}$ Para tanto, é necessário olhar com atenção à formação dos profissionais da saúde, acreditando que o sistema educacional exibe um papel significativo no desenvolvimento de currículos que permitam um aperfeiçoamento na comunicação profissionalusuário, a partir de um papel mais ativo frente às questões de saúde ${ }^{40}$ planejados numa perspectiva de que o letramento em saúde não se trata apenas de uma competência particular das pessoas, mas um, entre outros determinantes, que impactam na qualidade de vida das populações. ${ }^{34}$

Tal compreensão destaca a importância do protagonismo do indivíduo na busca de ferramentas capazes de proporcionar, melhorar e manter condições ideais para a manutenção da qualidade de vida e saúde, partindo de uma maior participação do sujeito no controle desse processo. Contudo, representa esforços não apenas individuais, mas coletivos e dos Serviços de Saúde na articulação de ações para a promoção da saúde e a autogestão do cuidado.

\section{CONCLUSÃO E IMPLICAÇÕES PARA A PRÁTICA}

A amostra se caracterizou por ser a maioria do sexo feminino, com baixa escolaridade e tempo de tratamento para as doenças crônicas acima de 10 anos. A maioria dos idosos participantes referiu ter hábito de leitura e compreender as 
informações repassadas pelos profissionais da equipe de saúde, no entanto houve prevalência de letramento funcional em saúde inadequado, seguido do limítrofe, entre os idosos portadores de doenças crônicas. A baixa escolaridade foi associada ao baixo desempenho no teste S-TOFHLA.

O letramento funcional em saúde inadequado nos idosos avaliados, muitas vezes, responsáveis pelo seu autocuidado, pode contribuir para agravos na condição de saúde e doença dessa população. Esse cenário chama atenção para a necessidade do estabelecimento de estratégias para capacitar os idosos e familiares para a gestão do seu próprio cuidado, compreendendo os requisitos necessários para a promoção da saúde e prevenção das doenças, sendo, portanto, relevante o reconhecimento deste determinante para o planejamento de ações que visem melhores resultados na produção do cuidado. Contudo, sugerese ampliar a amostra para confirmação dos achados, assim como a adaptação de instrumentos direcionados para avaliar o letramento funcional em saúde da população idosa brasileira.

\section{FINANCIAMENTO}

O presente trabalho foi realizado com apoio da Coordenação de Aperfeiçoamento de Pessoal de Nível Superior - Brasil (CAPES) - Código de Financiamento 001, Bolsa de mestrado concedido a Paulo Cassiano Simor dos Santos.

\section{CONTRIBUIÇÕES DOS AUTORES}

Desenho do estudo. Helenice de Moura Scortegagna. Paulo Cassiano Simor dos Santos. Maria Izabel Penha de Oliveira Santos. Marilene Rodrigues Portella.

Coleta ou produção dos dados. Helenice de Moura Scortegagna. Paulo Cassiano Simor dos Santos. Maria Izabel Penha de Oliveira Santos. Marilene Rodrigues Portella.

Análise de dados. Helenice de Moura Scortegagna. Paulo Cassiano Simor dos Santos. Maria Izabel Penha de Oliveira Santos. Marilene Rodrigues Portella.

Interpretação dos resultados. Helenice de Moura Scortegagna. Paulo Cassiano Simor dos Santos. Maria Izabel Penha de Oliveira Santos. Marilene Rodrigues Portella.

Redação e revisão crítica do manuscrito. Helenice de Moura Scortegagna. Paulo Cassiano Simor dos Santos. Maria Izabel Penha de Oliveira Santos. Marilene Rodrigues Portella.

Aprovação da versão final do artigo. Helenice de Moura Scortegagna. Paulo Cassiano Simor dos Santos. Maria Izabel Penha de Oliveira Santos. Marilene Rodrigues Portella.

Responsabilidade por todos os aspectos do conteúdo e a integridade do artigo publicado. Helenice de Moura Scortegagna. Paulo Cassiano Simor dos Santos. Maria Izabel Penha de Oliveira Santos. Marilene Rodrigues Portella.

\section{EDITOR ASSOCIADO}

Sofia Sabina Lavado Huarcaya

\section{REFERÊNCIAS}

1. Banco Mundial. Envelhecendo em um Brasil mais velho: implicações do envelhecimento populacional para o crescimento econômico, a redução da pobreza, as finanças públicas e a prestação de serviços. [Internet] Washington:The World Bank; 2011 [citado 2020 jun 12]. Disponível em: https://pesquisa.bvsalud.org/bvsms/resource/pt/mis-31970

2. Miranda GMD, Mendes ACG, Silva ALA. Public policies challenges on the background of demographic transition and social changes in Brazil. Interface (Botucatu). 2017 jun;21(61):309-20. http://dx.doi. org/10.1590/1807-57622016.0136.

3. Ministério da Saúde (BR). Plano de ações estratégicas para o enfrentamento das doenças crônicas não transmissíveis (DCNT) no Brasil 2011-2022. [Internet]. Brasília (DF): Ministério da Saúde; 2011 [citado 2020 jun 12]. Disponível em: http://bvsms.saude.gov.br/bvs/ publicacoes/plano_acoes_enfrent_dcnt_2011.pdf

4. Instituto Brasileiro de Geografia e Estatística. Pesquisa nacional por amostra de domicílios: síntese de indicadores 2015/IBGE. [Internet]. Rio de Janeiro: IBGE; 2016 [citado 2020 jun 12]. Disponível em: https:// biblioteca.ibge.gov.br/visualizacao/livros/liv94935.pdf

5. Instituto Paulo Montenegro. Ação Educativa. INAF Brasil 2018: resultados preliminares. [Internet]. São Paulo: Ação Educativa/IPM; 2018 [citado 2020 jun 12]. Disponível em: http://acaoeducativa.org.br/ wp-content/uploads/2018/08/Inaf2018_Relat\%C3\%B3rio-ResultadosPreliminares_v08Ago2018.pdf

6. Cavaco A, Santos AL. Avaliação da legibilidade de folhetos informativos e literácia em saúde. Rev Saude Publica. 2012 out;46(5):918-22. http:// dx.doi.org/10.1590/S0034-89102012000500019. PMid:23128269.

7. World Health Communication Associates. Health literacy: "The Basics" Revised Edition [Internet]. United Kingdom: World Health Communication Associates Ltd; 2011 [citado 2020 jun 12]. Disponível em: http://www. whcaonline.org/uploads/publications/HL-FINAL-14.7.2011-2.pdf

8. Maragno CAD. Associação entre letramento em saúde e adesão ao tratamento medicamentoso [tese]. Porto Alegre (RS): Programa de Pós-graduação em Ciências Farmacêuticas, Universidade Federal do Rio Grande do Sul; 2009.

9. Martins NFF, Abreu DPG, Silva BTD, Semedo DSDRC, Pelzer MT, lenczak FS. Functional health literacy and adherence to the medication in older adults: integrative review. Rev Bras Enferm. 2017 ago;70(4):868 74. http://dx.doi.org/10.1590/0034-7167-2016-0625. PMid:28793120.

10. Volpato LF, Martins LC, Mialhe FL. Bulas de medicamentos e profissionais de saúde: ajudam ou complicam a compreensão dos usuários? Rev Cienc Farm Basica Apl. 2009;30(3):309-14.

11. World Health Organization. A conceptual framework for action on the social determinants of health: social determinants of health discussion Paper 2 [Internet]. Geneve: WHO; 2010 [citado 2020 jun 12]. Disponível em: http://apps.who.int/iris/bitstream/10665/44489/1/9789241500852_eng. pdf

12. Commission on Social Determinants of Health. Closing the gap in a generation: health equity through action on the social determinants of health. Final Report of the Commission on Social Determinants of Health [Internet]. Geneva:WHO;2008 [citado 2020 jun 12]. Disponível em: https:// apps.who.int/iris/bitstream/handle/10665/43943/9789241563703_eng. pdf;jsess

13. Liu YB, Liu L, Li YF, Chen YL. Relationship between Health Literacy, Health-Related Behaviors and Health Status: A survey of elderly Chinese. Int J Environ Res Public Health. 2015;12(8):9714-25. http:// dx.doi.org/10.3390/ijerph120809714. PMid:26295246.

14. Poureslami I, Nimmon L, Rootman I, Fitzgerald MJ. Health literacy and chronic disease management: drawing from expert knowledge to set an agenda. Health Promot Int. 2017;32(4):743-54. http://dx.doi. org/10.1093/heapro/daw003. PMid:26873913.

15. Institute of Medicine. Health literacy: A prescription to end confusion. Washington, DC: The National Academies Press; 2004. https://doi. org/10.17226/10883.

16. Pleasant A, Rudd RE, O'Leary C, Paasche-Orlow MK, Allen MP, Alvarado-Little $\mathrm{W}$ et al. Considerations for a new definition of Health Literacy. Discussion Paper, National Academy of Medicine, Washington, 
DC: Academia Nacional de Medicina (NAM); 2016. https://doi. org/10.31478/201604a

17. Passamai MPB, Sampaio HAC, Lima JWO. Letramento funcional em adultos no contexto do sistema único de saúde [Internet]. Fortaleza: EdUECE; 2013. [citado 2020 jun 12]. Disponível em: https://siduece. uece.br/siduece/trabalhoAcademicoPublico.jsf?id=74409

18. Passamai MPB, Sampaio HAC, Dias AMI, Cabral LA. Letramento funcional em saúde: reflexões e conceitos sobre seu impacto na interação entre usuários, profissionais e sistema de saúde. Interface (Botucatu). 2012 jun;16(41):301-14. http://dx.doi.org/10.1590/S1414 32832012005000027.

19. Fontenelles MJ. Bioestatística aplicada à pesquisa experimental. São Paulo: Livraria da Física; 2012.

20. Ministério da Saúde (BR). Secretaria de Atenção à Saúde. Departamento de Atenção Básica. Envelhecimento e saúde da pessoa idosa [Internet] Brasília: Ministério da Saúde; 2007 [citado 2020 jun 12]. Disponível em: http://bvsms.saude.gov.br/bvs/publicacoes/abcad19.pdf

21. Baker DW, Williams MV, Parker RM, Gazmararian JA, Nurss J. Development of a brief test to measure functional health literacy. Patient Educ Couns. 1999 set;38(1):33-42. http://dx.doi.org/10.1016/S07383991(98)00116-5. PMid:14528569.

22. Carthery-Goulart MT, Anghinah R, Areza-Fegyveres R, Bahia VS, Brucki SMD, Damin A et al. Performance of a Brazilian population on the test of functional health literacy in adults. Rev Saude Publica. 2009 ago;43(4):631-8. http://dx.doi.org/10.1590/S0034-89102009005000031. PMid:19488667.

23. Carollo S. Low health literacy in older women: The influence of patientclinician relationships. Geriatr Nurs. 2015 mar-abr;36(2, Suppl.):38-42. http://dx.doi.org/10.1016/j.gerinurse.2015.02.017. PMid:25858518.

24. Grabowski CPR, Freitas JGA. Perfil e letramento funcional em saúde dos pacientes idosos hipertensos atendidos em unidade Escola Saúde da Família na região noroeste de Goiânia-Goiás. Resumo dos trabalhos publicados no II Congresso de Ciência e Tecnologia da PUC Goiás; 2016 out. 19-21; Goiânia (GO), Brasil. Goiânia, GO: PUC; 2016 Disponível em: http://pucgoias.edu.br/ucg/prope/pesquisa/anais/2016/ PDF/XVII_JORNADA_INICIACAO_CIENTIFICA/anais-2congressoct2016-inicientifica.297.pdf

25. Paskulin LMG, Aires M, Valer DB, de Morais EP, Freitas IBA. Adaptação de um instrumento que avalia alfabetização em saúde das pessoas idosas. Acta Paul Enferm. 2011;24(2):271-7. http://dx.doi.org/10.1590/ S0103-21002011000200018.

26. Rodrigues FFL, Santos MA, Teixeira CRS, Gonela JT, Zanetti ML. Relationship between knowledge, attitude, education and duration of disease in individuals with diabetes mellitus. Acta Paul Enferm 2012;25(2):284-90. http://dx.doi.org/10.1590/S0103-21002012000200020.

27. Federman $A D$, Wolf $M$, Sofianou $A$, Wilson $E A H$, Martynenko $M$, Halm EA et al. The association of health literacy with illness and medication beliefs among older adults with asthma. Patient Educ Couns. 2013 ago;92(2):273-8. http://dx.doi.org/10.1016/j.pec.2013.02.013. PMid:23523196.
28. Sarkar U, Karter AJ, Liu JY, Moffet HH, Adler NE, Schillinger D. Hypoglycemia is more common among type 2 diabetes patients with limited health literacy: the Diabetes Study of Northern California (DISTANCE). J Gen Intern Med. 2010;25(9):962-8. http://dx.doi.org/10.1007/s11606-0101389-7. PMid:20480249.

29. Souza JG, Apolinario D, Magaldi RM, Busse AL, Campora F, Jacob-Filho W. Functional health literacy and glycaemic control in older adults with type 2 diabetes: a cross-sectional study. BMJ Open. 2014;4(2):e004180. http://dx.doi.org/10.1136/bmjopen-2013-004180. PMid:24525392.

30. Berkman ND, Sheridan SL, Donahue KE, Halpern DJ, Viera A, Crotty Ket al. Health literacy interventions and outcomes: an updated systematic review. Evid Rep Technol Assess (Full Rep). 2011 mar;(199):1-941. PMid:23126607.

31. Santos MIPO, Portella MR. Condições do letramento funcional em saúde de um grupo de idosos diabéticos. Rev Bras Enferm. 2016 fev;69(1):156-64. http://dx.doi.org/10.1590/0034-7167.2016690121i. PMid:26871229.

32. Chesser AK, Keene Woods N, Smothers K, Rogers N. Health Literacy and older adults: A Systematic Review. Gerontol Geriatr Med. 2016 jandez;2:2333721416630492. http://dx.doi.org/10.1177/2333721416630492. PMid:28138488.

33. Van Der Heide I, Rademakers J, Schipper M, Droomers M, Sørensen K Uiters E. Health literacy of Dutch adults: a cross sectional survey. BMC Public Health. 2013 fev;13(179):1-11. http://dx.doi.org/10.1186/1471 2458-13-179. PMid:23445541.

34. Mialhe FL, Carthery-Goulart MT. Letramento em saúde e promoção da saúde. In: Pelicioni MCF, Mialhe FL, organizadores. Educação e promoção da saúde: teoria e prática. $2^{2}$ ed. São Paulo: Santos; 2019 p. 133-180.

35. Marques SRL, Lemos SMA. Instrumentos de avaliação do letramento em saúde: revisão de literatura. Audiol Commun Res. 2017;22(0):e1757. http://dx.doi.org/10.1590/2317-6431-2016-1757.

36. Silveira VC, Paskulin LMG. Perfil e rede de apoio de idosos internados na emergência do Hospital das Clínicas de Porto Alegre. Estud interdiscipl envelhec. 2014;19(2):377-396.

37. Coleman CA, Fromer A. A health literacy training intervention for physicians and other health professionals. Fam Med. 2015 maio;47(5):388-92. PMid:25905883.

38. Hadden KB. Health literacy training for health professions students Patient Educ Couns. 2015;98(7):918-20. http://dx.doi.org/10.1016/j. pec.2015.03.016. PMid:25850755

39. Lambert M, Luke J, Downey B, Crengle S, Kelaher M, Reid S et al. Health literacy: health professionals' understandings and their perceptions of barriers that Indigenous patients encounter. BMC Health Serv Res. 2014 nov;14(614):1-10. http://dx.doi.org/10.1186/s12913-014-0614-1. PMid:25471387.

40. Coleman CA, Hudson S, Maine LL. Health literacy practices and educational competencies for health professionals: a consensus study. J Health Commun. 2013;18(Suppl. 1):82-102. http://dx.doi.org/10.108 0/10810730.2013.829538. PMid:24093348. 